Andrews University

Digital Commons @ Andrews University

Faculty Publications

10-1-2005

\title{
Multijet Production in Neutral Current Deep Inelastic Scattering at HERA and Determination of $\boldsymbol{\alpha}_{\mathrm{S}}$
}

\author{
S. Chekanov \\ Argonne National Laboratory \\ M. Derrick \\ Argonne National Laboratory \\ S. Magill \\ Argonne National Laboratory \\ S. Miglioranzi \\ Argonne National Laboratory \\ B. Musgrave \\ Argonne National Laboratory
}

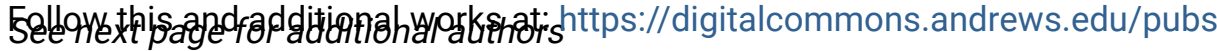

Part of the Physics Commons

\section{Recommended Citation}

Chekanov, S.; Derrick, M.; Magill, S.; Miglioranzi, S.; Musgrave, B.; Repond, J.; Yoshida, R.; Mattingly, Margarita C. K.; Pavel, N.; Yagües Molina, A. G.; Antonioli, P.; Bari, G.; Basile, M.; Bellagamba, L.; Boscherini, D.; Bruni, A.; Bruni, G.; Cara Romeo, G.; Cifarelli, L.; Cindolo, F.; Contin, A.; Corradi, M.; de Pasquale, S.; Giusti, P.; Iacobucci, G.; Margotti, A.; Montanari, A.; Nania, R.; Palmonari, F.; Pesci, A.; and Polini, A., "Multijet Production in Neutral Current Deep Inelastic Scattering at HERA and Determination of $\alpha_{s}$ " (2005). Faculty Publications. 2073.

https://digitalcommons.andrews.edu/pubs/2073

This Article is brought to you for free and open access by Digital Commons @ Andrews University. It has been accepted for inclusion in Faculty Publications by an authorized administrator of Digital Commons @ Andrews University. For more information, please contact repository@andrews.edu. 


\section{Authors}

S. Chekanov, M. Derrick, S. Magill, S. Miglioranzi, B. Musgrave, J. Repond, R. Yoshida, Margarita C. K. Mattingly, N. Pavel, A. G. Yagües Molina, P. Antonioli, G. Bari, M. Basile, L. Bellagamba, D. Boscherini, A. Bruni, G. Bruni, G. Cara Romeo, L. Cifarelli, F. Cindolo, A. Contin, M. Corradi, S. de Pasquale, P. Giusti, G. lacobucci, A. Margotti, A. Montanari, R. Nania, F. Palmonari, A. Pesci, and A. Polini 


\section{Multijet production in neutral current deep inelastic scattering at HERA and determination of $\alpha_{s}$}

The ZEUS Collaboration

S. Chekanov, M. Derrick, S. Magill, S. Miglioranzi ${ }^{1}$, B. Musgrave, J. Repond, R. Yoshida

Argonne National Laboratory, Argonne, Illinois 60439-4815, USA ${ }^{\mathrm{n}}$

M.C.K. Mattingly

Andrews University, Berrien Springs, Michigan 49104-0380, USA

N. Pavel, A.G. Yagües Molina

Institut für Physik der Humboldt-Universität zu Berlin, Berlin, Germany

P. Antonioli, G. Bari, M. Basile, L. Bellagamba, D. Boscherini, A. Bruni, G. Bruni, G. Cara Romeo, L. Cifarelli,

F. Cindolo, A. Contin, M. Corradi, S. De Pasquale, P. Giusti, G. Iacobucci, A. Margotti, A. Montanari, R. Nania,

F. Palmonari, A. Pesci, A. Polini, L. Rinaldi, G. Sartorelli, A. Zichichi

University and INFN Bologna, Bologna, Italy ${ }^{\mathrm{e}}$

G. Aghuzumtsyan, D. Bartsch, I. Brock, S. Goers, H. Hartmann, E. Hilger, P. Irrgang, H.-P. Jakob, O. Kind, U. Meyer, E. Paul ${ }^{2}$, J. Rautenberg, R. Renner, K.C. Voss ${ }^{3}$, M. Wang, M. Wlasenko

Physikalisches Institut der Universität Bonn, Bonn, Germany ${ }^{\mathrm{b}}$

D.S. Bailey ${ }^{4}$, N.H. Brook, J.E. Cole, G.P. Heath, T. Namsoo, S. Robins

H.H. Wills Physics Laboratory, University of Bristol, Bristol, United Kingdom ${ }^{\mathrm{m}}$

M. Capua, A. Mastroberardino, M. Schioppa, G. Susinno, E. Tassi

Calabria University, Physics Department and INFN, Cosenza, Italy

J.Y. Kim, K.J. Ma ${ }^{5}$

Chonnam National University, Kwangju, South Korea ${ }^{\mathrm{g}}$

M. Helbich, Y. Ning, Z. Ren, W.B. Schmidke, F. Sciulli

Nevis Laboratories, Columbia University, Irvington on Hudson, New York $10027^{\circ}$

J. Chwastowski, A. Eskreys, J. Figiel, A. Galas, K. Olkiewicz, P. Stopa, D. Szuba, L. Zawiejski

Institute of Nuclear Physics, Cracow, Poland ${ }^{i}$

L. Adamczyk, T. Bołd, I. Grabowska-Bołd, D. Kisielewska, A.M. Kowal, J. Łukasik, M. Przybycień, L. Suszycki, J. Szuba ${ }^{6}$

Faculty of Physics and Applied Computer Science, AGH-University of Science and Technology, Cracow, Poland ${ }^{\mathrm{P}}$

A. Kotański ${ }^{7}$, W. Słomiński

Department of Physics, Jagellonian University, Cracow, Poland

V. Adler, U. Behrens, I. Bloch, K. Borras, G. Drews, J. Fourletova, A. Geiser, D. Gladkov, P. Göttlicher ${ }^{8}$, O. Gutsche, T. Haas, W. Hain, C. Horn, B. Kahle, U. Kötz, H. Kowalski, G. Kramberger, D. Lelas ${ }^{9}$, H. Lim, B. Löhr, R. Mankel, I.-A. Melzer-Pellmann, C.N. Nguyen, D. Notz, A.E. Nuncio-Quiroz, A. Raval, R. Santamarta, U. Schneekloth, U. Stösslein, G. Wolf, C. Youngman, W. Zeuner

Deutsches Elektronen-Synchrotron DESY, Hamburg, Germany

S. Schlenstedt

Deutsches Elektronen-Synchrotron DESY, Zeuthen, Germany

G. Barbagli, E. Gallo, C. Genta, P. G. Pelfer

University and INFN, Florence, Italy ${ }^{\mathrm{e}}$

A. Bamberger, A. Benen, F. Karstens, D. Dobur, N.N. Vlasov ${ }^{10}$

Fakultät für Physik der Universität Freiburg i.Br., Freiburg i.Br., Germany ${ }^{\mathrm{b}}$ 
P.J. Bussey, A.T. Doyle, J. Ferrando, J. Hamilton, S. Hanlon, D.H. Saxon, I.O. Skillicorn

Department of Physics and Astronomy, University of Glasgow, Glasgow, United Kingdom ${ }^{\mathrm{m}}$

I. Gialas ${ }^{11}$

Department of Engineering in Management and Finance, Univ. of Aegean, Greece

T. Carli, T. Gosau, U. Holm, N. Krumnack ${ }^{12}$, E. Lohrmann, M. Milite, H. Salehi, P. Schleper, T. Schörner-Sadenius, S. Stonjek ${ }^{13}$, K. Wichmann, K. Wick, A. Ziegler, Ar. Ziegler

Hamburg University, Institute of Exp. Physics, Hamburg, Germany ${ }^{b}$

C. Collins-Tooth ${ }^{14}$, C. Foudas, C. Fry, R. Gonçalo ${ }^{15}$, K.R. Long, A.D. Tapper

Imperial College London, High Energy Nuclear Physics Group, London, United Kingdom ${ }^{\mathrm{m}}$

M. Kataoka ${ }^{16}$, K. Nagano, K. Tokushuku ${ }^{17}$, S. Yamada, Y. Yamazaki

Institute of Particle and Nuclear Studies, KEK, Tsukuba, Japan ${ }^{\mathrm{f}}$

A.N. Barakbaev, E.G. Boos, N.S. Pokrovskiy, B.O. Zhautykov

Institute of Physics and Technology of Ministry of Education and Science of Kazakhstan, Almaty, Kazakhstan

D. Son

Kyungpook National University, Center for High Energy Physics, Daegu, South Korea ${ }^{\mathrm{g}}$

J. de Favereau, K. Piotrzkowski

Institut de Physique Nucléaire, Université Catholique de Louvain, Louvain-la-Neuve, Belgium ${ }^{q}$

F. Barreiro, C. Glasman ${ }^{18}$, O. González, M. Jimenez, L. Labarga, J. del Peso, J. Terrón, M. Zambrana

Departamento de Física Teórica, Universidad Autónoma de Madrid, Madrid, Spain ${ }^{1}$

M. Barbi, F. Corriveau, C. Liu, S. Padhi, M. Plamondon, D.G. Stairs, R. Walsh, C. Zhou

Department of Physics, McGill University, Montréal, Québec, Canada H3A 2T8 ${ }^{\mathrm{a}}$

T. Tsurugai

Meiji Gakuin University, Faculty of General Education, Yokohama, Japan ${ }^{\mathrm{f}}$

A. Antonov, P. Danilov, B.A. Dolgoshein, V. Sosnovtsev, A. Stifutkin, S. Suchkov

Moscow Engineering Physics Institute, Moscow, Russia ${ }^{j}$

R.K. Dementiev, P.F. Ermolov, L.K. Gladilin, I.I. Katkov, L.A. Khein, I.A. Korzhavina, V.A. Kuzmin, B.B. Levchenko, O.Yu. Lukina, A.S. Proskuryakov, L.M. Shcheglova, D.S. Zotkin, S.A. Zotkin

Moscow State University, Institute of Nuclear Physics, Moscow, Russiak

I. Abt, C. Büttner, A. Caldwell, X. Liu, J. Sutiak

Max-Planck-Institut für Physik, München, Germany

N. Coppola, G. Grigorescu, S. Grijpink, A. Keramidas, E. Koffeman, P. Kooijman, E. Maddox, A. Pellegrino,

S. Schagen, H. Tiecke, M. Vázquez, L. Wiggers, E. de Wolf

NIKHEF and University of Amsterdam, Amsterdam, Netherlands ${ }^{\mathrm{h}}$

N. Brümmer, B. Bylsma, L.S. Durkin, T.Y. Ling

Physics Department, Ohio State University, Columbus, Ohio $43210^{\mathrm{n}}$

P.D. Allfrey, M.A. Bell, A.M. Cooper-Sarkar, A. Cottrell, R.C.E. Devenish, B. Foster, G. Grzelak, C. Gwenlan ${ }^{19}$, T. Kohno, S. Patel, P.B. Straub, R. Walczak

Department of Physics, University of Oxford, Oxford United Kingdom ${ }^{\mathrm{m}}$

P. Bellan, A. Bertolin, R. Brugnera, R. Carlin, R. Ciesielski, F. Dal Corso, S. Dusini, A. Garfagnini, S. Limentani,

A. Longhin, L. Stanco, M. Turcato

Dipartimento di Fisica dell' Università and INFN, Padova, Italy ${ }^{\mathrm{e}}$

E.A. Heaphy, F. Metlica, B.Y. Oh, J.J. Whitmore ${ }^{20}$

Department of Physics, Pennsylvania State University, University Park, Pennsylvania $16802^{\circ}$

Y. Iga

Polytechnic University, Sagamihara, Japan ${ }^{\mathrm{f}}$

G. D’Agostini, G. Marini, A. Nigro

Dipartimento di Fisica, Università 'La Sapienza' and INFN, Rome, Italy ${ }^{\mathrm{e}}$

J.C. Hart

Rutherford Appleton Laboratory, Chilton, Didcot, Oxon, United Kingdom ${ }^{\mathrm{m}}$ 
H. Abramowicz ${ }^{21}$, A. Gabareen, S. Kananov, A. Kreisel, A. Levy

Raymond and Beverly Sackler Faculty of Exact Sciences, School of Physics, Tel-Aviv University, Tel-Aviv, Israel ${ }^{\mathrm{d}}$

M. Kuze

Department of Physics, Tokyo Institute of Technology, Tokyo, Japan ${ }^{\mathrm{f}}$

S. Kagawa, T. Tawara

Department of Physics, University of Tokyo, Tokyo, Japan ${ }^{\mathrm{f}}$

R. Hamatsu, H. Kaji, S. Kitamura ${ }^{22}$, K. Matsuzawa, O. Ota, Y.D. Ri

Tokyo Metropolitan University, Department of Physics, Tokyo, Japan ${ }^{\mathrm{f}}$

M. Costa, M.I. Ferrero, V. Monaco, R. Sacchi, A. Solano

Università di Torino and INFN, Torino, Italy ${ }^{\mathrm{e}}$

M. Arneodo, M. Ruspa

Università del Piemonte Orientale, Novara, and INFN, Torino, Italy ${ }^{\mathrm{e}}$

S. Fourletov, T. Koop, J.F. Martin, A. Mirea

Department of Physics, University of Toronto, Toronto, Ontario, Canada M5S 1A7 ${ }^{\mathrm{a}}$

J.M. Butterworth ${ }^{23}$, R. Hall-Wilton, T.W. Jones, J.H. Loizides ${ }^{24}$, M.R. Sutton ${ }^{4}$, C. Targett-Adams, M. Wing Physics and Astronomy Department, University College London, London, United Kingdom ${ }^{\mathrm{m}}$

J. Ciborowski ${ }^{25}$, P. Kulinski, P. Łużniak ${ }^{26}$, J. Malka ${ }^{26}$, R.J. Nowak, J.M. Pawlak, J. Sztuk ${ }^{27}$, T. Tymieniecka, A. Tyszkiewicz ${ }^{26}$, A. Ukleja, J. Ukleja ${ }^{28}$, A.F. Żarnecki

Warsaw University, Institute of Experimental Physics, Warsaw, Poland

M. Adamus, P. Plucinski

Institute for Nuclear Studies, Warsaw, Poland

Y. Eisenberg, D. Hochman, U. Karshon, M.S. Lightwood

Department of Particle Physics, Weizmann Institute, Rehovot, Israel ${ }^{\mathrm{c}}$

A. Everett, D. Kçira, S. Lammers, L. Li, D.D. Reeder, M. Rosin, P. Ryan, A.A. Savin, W.H. Smith

Department of Physics, University of Wisconsin, Madison, Wisconsin 53706, USA ${ }^{\mathrm{n}}$

S. Dhawan

Department of Physics, Yale University, New Haven, Connecticut 06520-8121, USA ${ }^{\mathrm{n}}$

S. Bhadra, C.D. Catterall, Y. Cui, G. Hartner, S. Menary, U. Noor, M. Soares, J. Standage, J. Whyte

Department of Physics, York University, Ontario, Canada M3J 1P3 ${ }^{a}$

1 also affiliated with University College London, UK

2 retired

3 now at the University of Victoria, British Columbia, Canada

4 PPARC Advanced fellow

${ }^{5}$ supported by a scholarship of the World Laboratory Björn Wiik Research Project

${ }^{6}$ partly supported by Polish Ministry of Scientific Research and Information Technology, grant no.2P03B 12625

7 supported by the Polish State Committee for Scientific Research, grant no. 2 P03B 09322

8 now at DESY group FEB, Hamburg, Germany

9 now at LAL, Université de Paris-Sud, IN2P3-CNRS, Orsay, France

10 partly supported by Moscow State University, Russia

11 also affiliated with DESY

12 now at Baylor University, USA

13 now at University of Oxford, UK

14 now at the Department of Physics and Astronomy, University of Glasgow, UK

15 now at Royal Holloway University of London, UK

16 also at Nara Women's University, Nara, Japan

17 also at University of Tokyo, Japan

18 Ramón y Cajal Fellow

19 PPARC Postdoctoral Research Fellow

20 on leave of absence at The National Science Foundation, Arlington, VA, USA

21 also at Max Planck Institute, Munich, Germany, Alexander von Humboldt Research Award

22 present address: Tokyo Metropolitan University of Health Sciences, Tokyo 116-8551, Japan

23 also at University of Hamburg, Germany, Alexander von Humboldt Fellow

24 partially funded by DESY 
25 also at Łódź University, Poland

26 Łódź University, Poland

27 Łódź University, Poland, supported by the KBN grant 2P03B12925

28 supported by the KBN grant 2P03B12725

Received: 1 April 2005 / Revised version: 28 June 2005 /

Published online: 30 August 2005 - (C) Springer-Verlag / Società Italiana di Fisica 2005

\begin{abstract}
Multijet production rates in neutral current deep inelastic scattering have been measured in the range of exchanged boson virtualities $10<Q^{2}<5000 \mathrm{GeV}^{2}$. The data were taken at the ep collider HERA with centre-of-mass energy $\sqrt{s}=318 \mathrm{GeV}$ using the ZEUS detector and correspond to an integrated luminosity of $82.2 \mathrm{pb}^{-1}$. Jets were identified in the Breit frame using the $k_{T}$ cluster algorithm in the longitudinally invariant inclusive mode. Measurements of differential dijet and trijet cross sections are presented as functions of jet transverse energy $\left(E_{T, B}^{\text {jet }}\right)$, pseudorapidity $\left(\eta_{\mathrm{LAB}}^{\text {jet }}\right)$ and $Q^{2}$ with $E_{T, B}^{\text {jet }}>5 \mathrm{GeV}$ and $-1<\eta_{\mathrm{LAB}}^{\text {jet }}<2.5$. Next-to-leading-order QCD calculations describe the data well. The value of the strong coupling constant $\alpha_{s}\left(M_{Z}\right)$, determined from the ratio of the trijet to dijet cross sections, is $\alpha_{s}\left(M_{Z}\right)=0.1179 \pm 0.0013$ (stat. $)_{-0.0046}^{+0.0028}$ (exp. $)_{-0.0046}^{+0.0064}$ (th.).
\end{abstract}

a supported by the Natural Sciences and Engineering Research Council of Canada (NSERC)

b supported by the German Federal Ministry for Education and Research (BMBF), under contract numbers HZ1GUA 2, HZ1GUB 0, HZ1PDA 5, HZ1VFA 5

c supported in part by the MINERVA Gesellschaft für Forschung GmbH, the Israel Science Foundation (grant no. 293/02-11.2), the U.S.-Israel Binational Science Foundation and the Benozyio Center for High Energy Physics

d supported by the German-Israeli Foundation and the Israel Science Foundation

e supported by the Italian National Institute for Nuclear Physics (INFN)

${ }^{\mathrm{f}}$ supported by the Japanese Ministry of Education, Culture, Sports, Science and Technology (MEXT) and its grants for Scientific Research

g supported by the Korean Ministry of Education and Korea Science and Engineering Foundation

$\mathrm{h}$ supported by the Netherlands Foundation for Research on Matter (FOM)

i supported by the Polish State Committee for Scientific Research, grant no. 620/E-77/SPB/DESY/P-03/DZ 117/20032005 and grant no. 1P03B07427/2004-2006

$\mathrm{j}$ partially supported by the German Federal Ministry for Education and Research (BMBF)

k supported by RF Presidential grant N 1685.2003.2 for the leading scientific schools and by the Russian Ministry of Education and Science through its grant for Scientific Research on High Energy Physics

${ }^{1}$ supported by the Spanish Ministry of Education and Science through funds provided by CICYT

${ }^{m}$ supported by the Particle Physics and Astronomy Research Council, UK

n supported by the US Department of Energy

o supported by the US National Science Foundation

p supported by the Polish Ministry of Scientific Research and Information Technology, grant no. 112/E-356/SPUB/DESY/P03/DZ 116/2003-2005 and 1 P03B 06527

q supported by FNRS and its associated funds (IISN and FRIA) and by an Inter-University Attraction Poles Programme subsidised by the Belgian Federal Science Policy Office

\section{Introduction}

Measurements of multijet production from initial-state hadrons and leptons have been carried out previously in collisions at the SPS $[1,2]$, the ISR $[3,4]$, the TEVATRON $[5,6]$ and at LEP $[7,8]$ as well as in photoproduction [9] and deep inelastic scattering (DIS) [10] at HERA. Multijet production in DIS at HERA has been used to test the predictions of perturbative QCD (pQCD) calculations over a large range of four-momentum transfer squared, $Q^{2}[11]$. Recently, the ZEUS and $\mathrm{H} 1$ collaborations have determined the strong coupling constant $\alpha_{s}$ from a variety of measurements of jet production and jet properties in both DIS [10,12-18] and photoproduction [19].

At leading order $(\mathrm{LO})$ in $\alpha_{s}$, dijet production in neutral current DIS proceeds via the boson-gluon-fusion (BGF, $V^{*} g \rightarrow q \bar{q}$ with $V=\gamma, Z^{0}$ ) and QCD-Compton (QCDC, $\left.V^{*} q \rightarrow q g\right)$ processes. Events with three jets can be seen as dijet processes with an additional gluon radiation or splitting of a gluon into a quark-antiquark pair and are directly sensitive to $\mathcal{O}\left(\alpha_{s}^{2}\right)$ QCD effects. The higher sensitivity to $\alpha_{s}$ and the large number of degrees of freedom of the trijet final state allow detailed testing of QCD predictions.

In the present analysis, the differential cross sections for the trijet production have been measured with high statistical precision. Measurements of the inclusive trijet cross section as a function of $Q^{2}$ and the jet transverse energy, $E_{T, B}^{\mathrm{jet}}$, in the Breit frame and the jet pseudorapidity, $\eta_{\mathrm{LAB}}^{\text {jet }}$, in the laboratory frame are presented. Predictions of pQCD at next-to-leading order (NLO) are compared to the measurements. In addition, the analysis includes the first $\alpha_{s}$ determination using the cross-section ratio of trijet to dijet production, $R_{3 / 2}$, at HERA. In this ratio, correlated experimental and theoretical uncertainties cancel, allowing for an extension of the measurement to low $Q^{2}$.

\section{Experimental set-up}

The data used in this analysis were collected during the 1998-2000 running period, when HERA operated with pro- 
tons of energy $E_{p}=920 \mathrm{GeV}$ and electrons or positrons ${ }^{1}$ of energy $E_{e}=27.5 \mathrm{GeV}$, and correspond to an integrated luminosity of $82.2 \pm 1.9 \mathrm{pb}^{-1}$. A detailed description of the ZEUS detector can be found elsewhere [20,21]. A brief outline of the components that are most relevant for this analysis is given below.

Charged particles are measured in the central tracking detector (CTD) [22-24], which operates in a magnetic field of $1.43 \mathrm{~T}$ provided by a thin superconducting solenoid. The CTD consists of 72 cylindrical drift chamber layers, organised in nine superlayers covering the polarangle ${ }^{2}$ region $15^{\circ}<\theta<164^{\circ}$. The transverse momentum resolution for full-length tracks can be parameterised as $\sigma\left(p_{T}\right) / p_{T}=0.0058 p_{T} \oplus 0.0065 \oplus 0.0014 / p_{T}$, with $p_{T}$ in $\mathrm{GeV}$. The tracking system was used to measure the interaction vertex with a typical resolution along (transverse to) the beam direction of $0.4(0.1) \mathrm{cm}$ and also to cross-check the energy scale of the calorimeter.

The high-resolution uranium-scintillator calorimeter (CAL) [25-28] covers $99.7 \%$ of the total solid angle and consists of three parts: the forward (FCAL), the barrel (BCAL) and the rear (RCAL) calorimeters. Each part is subdivided transversely into towers and longitudinally into one electromagnetic section (EMC) and either one (in RCAL) or two (in BCAL and FCAL) hadronic sections (HAC). The smallest subdivision of the calorimeter is called a cell. Under test-beam conditions, the CAL single-particle relative energy resolutions were $\sigma(E) / E=0.18 / \sqrt{E}$ for electrons and $\sigma(E) / E=0.35 / \sqrt{E}$ for hadrons, with $E$ in $\mathrm{GeV}$.

The luminosity was measured from the rate of the bremsstrahlung process $e p \rightarrow e \gamma p$. The resulting small angle energetic photons were measured by the luminosity monitor [29-31], a lead-scintillator calorimeter placed in the HERA tunnel at $Z=-107 \mathrm{~m}$.

\section{Kinematics and event selection}

A three-level trigger system was used to select events online $[21,32-34]$. Neutral current DIS events were selected by requiring that the scattered electron with energy more than $4 \mathrm{GeV}$ was measured in the CAL [35].

The offline kinematic variables $Q^{2}$ (four-momentum transfer squared), $x_{\mathrm{Bj}}$ (Bjorken scaling variable) and $y=$ $Q^{2} /\left(s x_{\mathrm{Bj}}\right)$ ( $s$ is the centre-of-mass energy squared) were reconstructed by the electron $(e)$, double angle (DA) [36] and Jacquet-Blondel (JB) [37] methods. The angle of the hadronic system, $\gamma_{\text {had }}$, corresponds, in the quark-parton model, to the direction of the scattered quark and was reconstructed from the CAL measurements of the hadronic final state.

\footnotetext{
1 In the following, the term "electron" denotes generically both the electron $\left(e^{-}\right)$and the positron $\left(e^{+}\right)$.

2 The ZEUS coordinate system is a right-handed Cartesian system, with the $Z$ axis pointing in the proton beam direction, referred to as the "forward direction", and the $X$ axis pointing left towards the centre of HERA. The coordinate origin is at the nominal interaction point.
}

If $\gamma_{\text {had }}$ was less than $90^{\circ}$ and the scattered-electron track could be well reconstructed by the CTD, the DA method was used; otherwise, the electron method was used. The offline selection of DIS events was similar to that used in a previous ZEUS measurement [38] and was based on the following requirements:

- $E_{e}^{\prime}>10 \mathrm{GeV}$, where $E_{e}^{\prime}$ is the scattered-electron energy after correction for energy loss in inactive material in front of the CAL, to achieve a high-purity sample of DIS events;

- $y_{e}<0.6$, where $y_{e}$ is $y$ reconstructed by the electron method, to reduce the photoproduction background;

$-y_{\mathrm{JB}}>0.04$, where $y_{\mathrm{JB}}$ is $y$ reconstructed by the JB method, to ensure sufficient accuracy for the DA reconstruction of $Q^{2}$;

- $\cos \gamma_{\text {had }}<0.7$, to ensure good reconstruction of jets in the Breit frame;

- $40<\sum_{i}\left(E-P_{Z}\right)_{i}<60 \mathrm{GeV}$, where the sum runs over all CAL energy deposits. The lower cut removed background from photoproduction and events with large initial-state QED radiation. The higher cut removed cosmic-ray background;

- $\left|Z_{\text {vertex }}\right|<50 \mathrm{~cm}$, where $Z_{\text {vertex }}$ is the reconstructed primary vertex $Z$-position, to select events consistent with ep collisions;

- $|X|>13$ or $|Y|>7 \mathrm{~cm}$, where $X$ and $Y$ are the impact positions of the scattered electron on the RCAL, to avoid the low-acceptance region adjacent to the rear beampipe.

The kinematic range of the analysis is defined as:

$$
10<Q^{2}<5000 \mathrm{GeV}^{2} \text { and } 0.04<y<0.6 .
$$

Jets were reconstructed using the $k_{T}$ cluster algorithm [39] in the longitudinally invariant inclusive mode [40]. The jet search was conducted in the Breit frame [41,42]. For each event, the jet search was performed using a combination of track and CAL information, excluding the cells and the track associated with the scattered electron. The selected tracks and CAL clusters were treated as massless Energy Flow Objects (EFOs) [43]. The clustering of objects was done according to the Snowmass convention [44].

The jet phase space is defined by selection cuts on the jet pseudorapidity $\eta_{\mathrm{LAB}}^{\text {jet }}$ in the laboratory frame and on the jet transverse energy $E_{T, B}^{\text {jet }}$ in the Breit frame:

$$
-1<\eta_{\mathrm{LAB}}^{\mathrm{jet}}<2.5 \quad \text { and } \quad E_{T, B}^{\mathrm{jet}}>5 \mathrm{GeV} .
$$

Events with two (three) or more jets were selected by requiring the invariant mass of the two (three) highest $E_{T, B}^{\text {jet }}$ jets to be:

$$
M_{2 \text { jets(3jets) }}>25 \mathrm{GeV} .
$$

These requirements were necessary to ensure a reliable prediction of the cross sections at NLO (see Sect. 5).

After all cuts, 37089 events with two or more jets (dijets) and 13665 events with three or more jets (trijets) remained. 


\section{Monte Carlo simulation}

Monte Carlo (MC) simulations were used to correct the data for detector effects, inefficiencies of the event selection and that of the jet reconstruction, as well as for QED effects. Neutral current DIS events were generated using the ARIADNE 4.08 program [45] and the LEPTO 6.5 program [46] interfaced to Heracles 4.5.2 [47] via DJANgo 6.2.4 [48]. The HERACLES program includes QED effects up to $\mathcal{O}\left(\alpha_{\mathrm{EM}}^{2}\right)$. In case of ARIADNE, the QCD cascade is simulated using the colour-dipole model [49], whereas for LEPTO, the matrix elements plus parton shower model is used. Both models use the Lund string model [50], as implemented in JETSET $7.4[51,52]$, for hadronisation.

The ZEUS detector response was simulated with a program based on GEANT 3.13 [53]. The generated events were passed through the detector simulation, subjected to the same trigger requirements as the data, and processed by the same reconstruction and offline programs.

Measured distributions of kinematic variables are well described by both the ARIADNE and LEPTO MC models after reweighting in $Q^{2}$. The LEPTO simulation gives a better overall description of the $E_{T, B}^{\mathrm{jet}}$ and invariant mass distributions. Therefore, the events generated with the LEPTO program were used to determine the acceptance corrections. The events generated with ARIADNE were used to estimate the systematic uncertainty associated with the treatment of the parton shower.

\section{NLO QCD calculations}

The NLO calculations were carried out in the $\overline{\mathrm{MS}}$ scheme for five massless quark flavors with the program NLOJET [54] using CTEQ6 [55], CTEQ4 [56], MRST99 [57] and ZEUS-S [58] for the proton parton density functions (PDFs). NLOJET allows a computation of the trijet production cross sections to next-to-leading order, i.e. including all terms up to $\mathcal{O}\left(\alpha_{s}^{3}\right)$. It was checked that the LO and NLO calculations from NLOJET agree with those of DiSENT [59] at the 1-2\% level for the dijet cross sections $[33,34]$.

For comparison with the data, the CTEQ6 parameterisations of the proton PDFs were used and the renormalisation and factorisation scales were both chosen to be $\left(\bar{E}_{T}^{2}+Q^{2}\right) / 4$, where for dijets (trijets) $\bar{E}_{T}$ is the average $E_{T}$ of the two (three) highest $E_{T}$ jets in a given event. The strong coupling constant was set to the value used in the CTEQ6 analysis, $\alpha_{s}\left(M_{Z}\right)=0.1179$, and evolved according to the two-loop solution of the renormalisation group equation.

The NLO QCD predictions were corrected for hadronisation effects using a bin-by-bin procedure. Hadronisation correction factors were defined for each bin as the ratio of the parton- to hadron-level cross sections and were calculated using the LEPTO MC program. The correction factors $C_{\text {had }}$ were typically in the range $1.15-1.35$ for most of the phase space.

\section{Corrections and systematic uncertainties}

The jet transverse energy was corrected for energy losses in the inactive material in front of the CAL using the samples of MC simulated events [18]. The cross sections for jets of hadrons in bins of $Q^{2}, E_{T, B}^{\mathrm{jet}}$ and $\eta_{\mathrm{LAB}}^{\mathrm{jet}}$ were obtained by applying a bin-by-bin correction to the measured jet distributions using the LEPTO program. The corrections take into account the efficiency of the trigger, the selection criteria and the purity and efficiency of the jet reconstruction. The correction factors were within about $20 \%$ of unity for all bins. Additional corrections for QED effects, $C_{\mathrm{QED}}$, calculated using HERACLES, were applied to the measured cross sections, $\sigma_{\text {Born }}=\sigma_{\text {meas. }} \cdot C_{\mathrm{QED}}$.

A detailed study of the sources contributing to the systematic uncertainties of the measurements was performed $[33,34]$. The main sources contributing to the systematic uncertainties are listed below (typical values of the systematic uncertainties in the dijet cross section and cross-section ratio $R_{3 / 2}$ are indicated in parentheses):

- jet-pseudorapidity cut - a change of \pm 0.1 (corresponding to the resolution) in the $\eta_{\mathrm{LAB}}^{\text {jet }}$ cuts imposed on the jets in the laboratory frame for both data and MC simulated events $(1 \%, 1 \%)$;

- jet transverse energy and invariant mass cuts - $E_{T, B}^{\mathrm{jet}}$ and $M_{2 \text { jets }}\left(M_{3 \text { jets }}\right)$ were simultaneously varied by the corresponding resolution near the cuts for both data and $\mathrm{MC}$ simulated events. Along with the previous systematic check, this takes into account the effect of the remaining differences between the data and the MC simulation $(3 \%, 3 \%)$;

- use of different parton shower model - using ARIADNE instead of LEPTO to evaluate the acceptance corrections $(2 \%, 4 \%)$;

- the absolute energy scale of the $C A L$ - varying $E_{T, B}^{\mathrm{jet}}$ by its uncertainty of $\pm 1 \%(>10 \mathrm{GeV})$ and $\pm 3 \%(<10 \mathrm{GeV})$ for $\mathrm{MC}$ events [14] $(6 \%, 3.5 \%)$.

The systematic uncertainties not associated with the absolute energy scale of the CAL were added in quadrature to the statistical uncertainties and are shown on the figures as error bars. The uncertainty due to the absolute energy scale of the CAL is highly correlated from bin-to-bin and is shown separately as a shaded band. The total systematic uncertainty and the uncertainty due to the absolute energy scale are also shown in Tables 1-9.

The main contributions to the theoretical uncertainties of the NLO QCD predictions are:

- uncertainties in the proton PDFs, which were estimated by repeating the calculations using 40 additional sets obtained under different theoretical assumptions as part of the CTEQ6 release $(2.5 \%, 2 \%)$;

- uncertainties in the correction factors, $C_{\text {had }}$, which were estimated by using the ARIADNE program instead of LEPTO $(6 \%, 4 \%)$;

- uncertainties due to terms beyond $N L O$, which were estimated by varying both $\mu_{R}$ and $\mu_{F}$ between $\left(\bar{E}_{T}^{2}+\right.$ $\left.Q^{2}\right)$ and $\left(\bar{E}_{T}^{2}+Q^{2}\right) / 16(10 \%, 7 \%)$. The use of $\bar{E}_{T}^{2}$ or 
$Q^{2}$ as the scale gives results within these uncertainties except when $\bar{E}_{T}^{2}$ is used in the highest $Q^{2}$ bin.

The total theoretical uncertainty was obtained by adding in quadrature the individual uncertainties listed above.

\section{Results}

\subsection{Differential cross sections}

The differential trijet cross sections as functions of $E_{T, B}^{\text {jet }}$ are presented in Fig. 1 and in Tables 1-3. The three highest $E_{T, B}^{\mathrm{jet}}$ jets were ordered in $E_{T, B}^{\mathrm{jet}}\left(E_{T, B}^{\mathrm{jet}, 1}>E_{T, B}^{\mathrm{jet}, 2}>E_{T, B}^{\mathrm{jet}, 3}\right)$. The observed decrease of the cross section for the first jet towards small values of $E_{T, B}^{\mathrm{jet}}$ is caused by the $E_{T, B}^{\mathrm{jet}}$ ordering combined with the requirement that the second and third jet have $E_{T, B}^{\mathrm{jet}}>5 \mathrm{GeV}$. For the second jet, a similar but less pronounced effect is observed. The NLO predictions using NLOJET, corrected for hadronisation effects, are compared to the data in Fig. 1. The QCD predictions provide

\section{ZEUS}

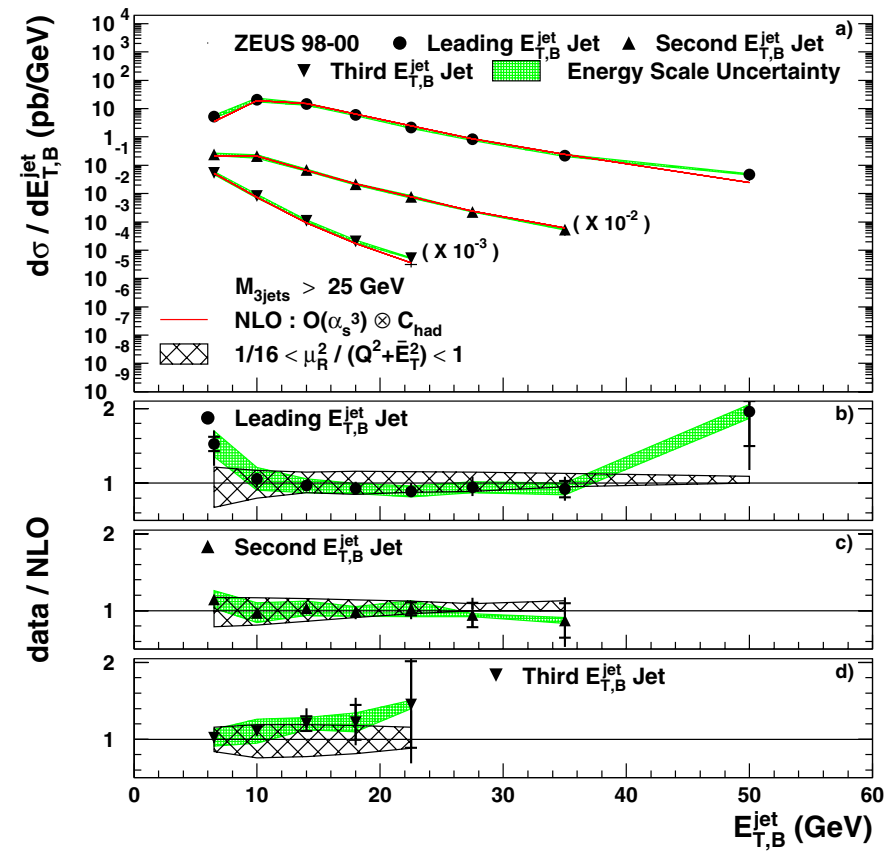

Fig. 1. a The inclusive trijet cross sections as functions of $E_{T, B}^{\text {jet }}$ with the jets ordered in $E_{T, B}^{\mathrm{jet}}$. The cross sections of the second and third jet were scaled by the factors shown for readability. The inner error bars represent the statistical uncertainties. The outer error bars represent the quadratic sum of statistical and systematic uncertainties not associated with the calorimeter energy scale. The shaded band indicates the calorimeter energy scale uncertainty. The predictions of perturbative QCD in next-to-leading order, corrected for hadronisation effects and using the CTEQ6 parameterisations of the proton PDFs, are compared to the data. $\mathbf{b}, \mathbf{c}$, and $\mathbf{d}$ show the ratio of the data to the predictions. The hatched band represents the renormalisation scale uncertainty of the QCD calculation
Table 1. The inclusive trijet cross section $d \sigma / d E_{T, B}^{\mathrm{jet}, 1}$ for jets of hadrons in the Breit frame, selected using the $k_{T}$ cluster algorithm in the longitudinally invariant inclusive mode. The statistical $\left(\delta_{\text {stat }}\right)$, systematic $\left(\delta_{\text {syst }}\right)$ and the absolute energy scale uncertainties $\left(\delta_{\mathrm{ES}}\right)$ are shown separately. The multiplicative correction factors for QED radiative effects $\left(C_{\mathrm{QED}}\right)$, applied to the data, and for hadronisation effects $\left(C_{\text {had }}\right)$, applied to the NLO predictions, are shown in the last two columns

\begin{tabular}{lclllll}
\hline $\begin{array}{l}E_{T, B}^{\text {jet, } 1} \\
(\mathrm{GeV})\end{array}$ & $\begin{array}{c}d \sigma / d E_{T, B}^{\text {jet, }, 1} \\
(\mathrm{pb} / \mathrm{GeV})\end{array}$ & $\delta_{\text {stat }}$ & $\delta_{\text {syst }}$ & $\delta_{\mathrm{ES}}$ & $C_{\mathrm{QED}}$ & $C_{\text {had }}$ \\
\hline $5-8$ & 5.22 & \pm 0.29 & ${ }_{-0.89}^{+0.75}$ & ${ }_{-0.50}^{+0.63}$ & 0.97 & 2.11 \\
$8-12$ & 20.2 & \pm 0.5 & ${ }_{-3.5}^{+3.0}$ & ${ }_{-2.9}^{+3.0}$ & 0.96 & 1.33 \\
$12-16$ & 14.4 & \pm 0.4 & ${ }_{-1.4}^{+1.6}$ & ${ }_{-1.3}^{+1.6}$ & 0.96 & 1.21 \\
$16-20$ & 6.05 & \pm 0.22 & ${ }_{-0.54}^{+0.68}$ & ${ }_{-0.44}^{+0.42}$ & 0.96 & 1.19 \\
$20-25$ & 2.19 & \pm 0.11 & ${ }_{-0.21}^{+0.32}$ & ${ }_{-0.16}^{+0.20}$ & 0.96 & 1.19 \\
$25-30$ & 0.828 & \pm 0.068 & ${ }_{-0.10}^{+0.12}$ & ${ }_{-0.065}^{+0.063}$ & 0.97 & 1.22 \\
$30-40$ & 0.222 & \pm 0.027 & ${ }_{-0.026}^{+0.036}$ & ${ }_{-0.017}^{+0.021}$ & 0.97 & 1.20 \\
$40-60$ & 0.047 & \pm 0.012 & ${ }_{-0.012}^{+0.004}$ & ${ }_{-0.004}^{+0.001}$ & 1.07 & 1.31 \\
\hline
\end{tabular}

Table 2. The inclusive trijet cross section $d \sigma / d E_{T, B}^{\mathrm{jet}, 2}$ for jets of hadrons in the Breit frame, selected using the $k_{T}$ cluster algorithm in the longitudinally invariant inclusive mode. Other details are as in the caption to Table 1

\begin{tabular}{lllllll}
\hline $\begin{array}{l}E_{T, B}^{\text {jet, }} \\
(\mathrm{GeV})\end{array}$ & $\begin{array}{l}d \sigma / d E_{T, B}^{\text {jet, }} \\
(\mathrm{pb} / \mathrm{GeV})\end{array}$ & $\delta_{\text {stat }}$ & $\delta_{\text {syst }}$ & $\delta_{\mathrm{ES}}$ & $C_{\mathrm{QED}}$ & $C_{\text {had }}$ \\
\hline $5-8$ & 24.0 & \pm 0.6 & ${ }_{-2.6}^{+2.6}$ & ${ }_{-2.4}^{+2.5}$ & 0.96 & 1.48 \\
$8-12$ & 20.7 & \pm 0.4 & ${ }_{-2.8}^{+3.0}$ & ${ }_{-2.6}^{+3.0}$ & 0.97 & 1.22 \\
$12-16$ & 6.70 & \pm 0.24 & ${ }_{-0.66}^{+0.65}$ & ${ }_{-0.56}^{+0.65}$ & 0.96 & 1.28 \\
$16-20$ & 2.16 & \pm 0.13 & ${ }_{-0.22}^{+0.14}$ & ${ }_{-0.14}^{+0.13}$ & 0.95 & 1.32 \\
$20-25$ & 0.780 & \pm 0.067 & ${ }_{-0.10}^{+0.087}$ & ${ }_{-0.070}^{+0.086}$ & 0.97 & 1.34 \\
$25-30$ & 0.225 & \pm 0.038 & ${ }_{-0.005}^{+0.034}$ & ${ }_{-0.005}^{+0.005}$ & 0.97 & 1.40 \\
$30-40$ & 0.054 & \pm 0.014 & ${ }_{-0.006}^{+0.013}$ & ${ }_{-0.001}^{+0.004}$ & 1.02 & 1.51 \\
\hline
\end{tabular}

Table 3. The inclusive trijet cross section $d \sigma / d E_{T, B}^{\mathrm{jet}, 3}$ for jets of hadrons in the Breit frame, selected using the $k_{T}$ cluster algorithm in the longitudinally invariant inclusive mode. Other details are as in the caption to Table 1

\begin{tabular}{lllllll}
\hline $\begin{array}{l}E_{T, B}^{\text {jet,3 }} \\
(\mathrm{GeV})\end{array}$ & $\begin{array}{c}d \sigma / d E_{T, B}^{\text {jet,3 }} \\
(\mathrm{pb} / \mathrm{GeV})\end{array}$ & $\delta_{\text {stat }}$ & $\delta_{\text {syst }}$ & $\delta_{\mathrm{ES}}$ & $C_{\mathrm{QED}}$ & $C_{\text {had }}$ \\
\hline $5-8$ & 52.8 & \pm 0.8 & ${ }_{-6.2}^{+6.3}$ & ${ }_{-5.3}^{+6.1}$ & 0.96 & 1.28 \\
$8-12$ & 8.06 & \pm 0.25 & ${ }_{-1.1}^{+1.1}$ & ${ }_{-1.1}^{+1.1}$ & 0.96 & 1.49 \\
$12-16$ & 1.11 & \pm 0.09 & ${ }_{-0.08}^{+0.17}$ & ${ }_{-0.08}^{+0.07}$ & 0.97 & 1.66 \\
$16-20$ & 0.208 & \pm 0.039 & ${ }_{-0.035}^{+0.025}$ & ${ }_{-0.018}^{+0.025}$ & 0.98 & 1.77 \\
$20-25$ & 0.052 & \pm 0.020 & ${ }_{-0.019}^{+0.005}$ & ${ }_{-0.003}^{+0.000}$ & 0.94 & 1.77 \\
\hline
\end{tabular}

a good description of both the shape and magnitude of the measured cross sections, even at low $E_{T, B}^{\mathrm{jet}}$.

Figure 2 and Tables $4-6$ show the differential trijet cross sections as functions of $\eta_{\mathrm{LAB}}^{\text {jet }}$. The three highest $E_{T, B}^{\text {jet }}$ jets were ordered in $\eta_{\mathrm{LAB}}^{\text {jet }}\left(\eta_{\mathrm{LAB}}^{\text {jet, }}>\eta_{\mathrm{LAB}}^{\text {jet, } 2}>\eta_{\mathrm{LAB}}^{\text {jet, } 3}\right)$. Figure 3 and Tables 7 and 8 show both the differential dijet and trijet cross section as functions of $Q^{2}$. In Figs. 2 and 3, the data 


\section{ZEUS}

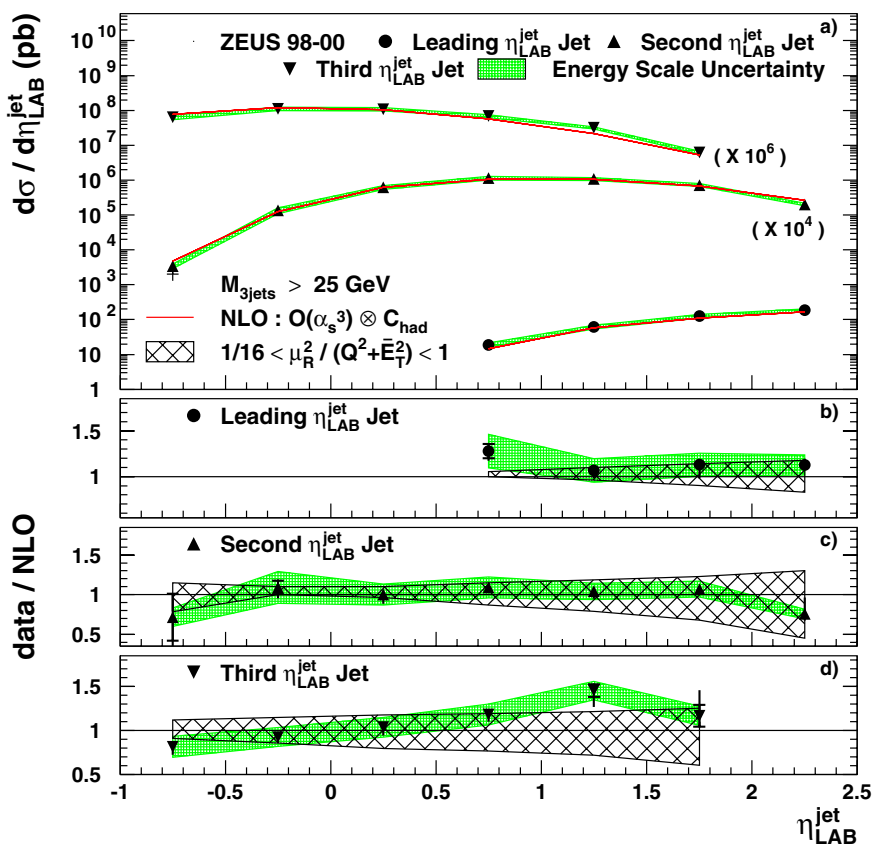

Fig. 2. a The inclusive trijet cross sections as functions of $\eta_{\mathrm{LAB}}^{\text {jet }}$ with the jets ordered in $\eta_{\mathrm{LAB}}^{\text {jet }}$. The cross sections of the second and third jet were scaled up for readability only. The predictions of perturbative QCD in next-to-leading order are compared to the data. $\mathbf{b}, \mathbf{c}$, and $\mathbf{d}$ show the ratio of the data to the predictions. Other details are as in the caption to Fig. 1

\section{ZEUS}

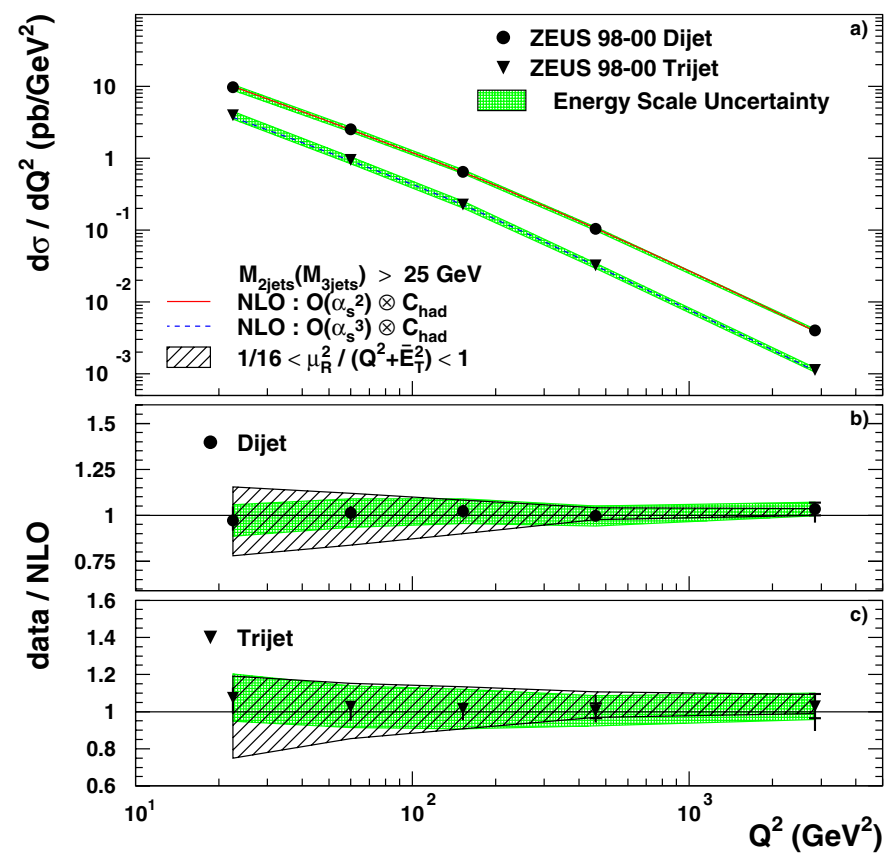

Fig. 3. a The inclusive dijet and trijet cross sections as functions of $Q^{2}$. The predictions of perturbative QCD in next-to-leading order are compared to the data. $\mathbf{b}$ and $\mathbf{c}$ show the ratio of the data to the predictions. Other details are as in the caption to Fig. 1
Table 4. The inclusive trijet cross section $d \sigma / d \eta_{\mathrm{LAB}}^{\mathrm{jet}, 1}$ for jets of hadrons in the Breit frame, selected using the $k_{T}$ cluster algorithm in the longitudinally invariant inclusive mode. Other details are as in the caption to Table 1

\begin{tabular}{lllllll}
\hline$\eta_{\mathrm{LAB}}^{\text {jet, }}$ & $\begin{array}{c}d \sigma / d \eta_{\mathrm{LAB}}^{\text {jet, }} \\
(\mathrm{pb})\end{array}$ & $\delta_{\text {stat }}$ & $\delta_{\text {syst }}$ & $\delta_{\mathrm{ES}}$ & $C_{\mathrm{QED}}$ & $C_{\mathrm{had}}$ \\
\hline $0.5-1.0$ & 19.1 & \pm 1.2 & ${ }_{-2.7}^{+2.9}$ & ${ }_{-2.6}^{+2.9}$ & 0.95 & 2.18 \\
$1.0-1.5$ & 61.2 & \pm 2.0 & ${ }_{-8.7}^{+7.3}$ & ${ }_{-7.3}^{+7.3}$ & 0.96 & 1.51 \\
$1.5-2.0$ & 125 & \pm 3 & ${ }_{-20}^{+15}$ & ${ }_{-13}^{+15}$ & 0.96 & 1.31 \\
$2.0-2.5$ & 186 & \pm 4 & ${ }_{-20}^{+23}$ & ${ }_{-18}^{+20}$ & 0.96 & 1.17 \\
\hline
\end{tabular}

Table 5. The inclusive trijet cross section $d \sigma / d \eta_{\mathrm{LAB}}^{\mathrm{jet}, 2}$ for jets of hadrons in the Breit frame, selected using the $k_{T}$ cluster algorithm in the longitudinally invariant inclusive mode. Other details are as in the caption to Table 1

\begin{tabular}{lclllll}
\hline$\eta_{\mathrm{LAB}}^{\text {jet,2 }}$ & $\begin{array}{c}d \sigma / d \eta_{\mathrm{LAB}}^{\text {jet,2 }} \\
(\mathrm{pb})\end{array}$ & $\delta_{\text {stat }}$ & $\delta_{\text {syst }}$ & $\delta_{\mathrm{ES}}$ & $C_{\mathrm{QED}}$ & $C_{\text {had }}$ \\
\hline$-1.0-0.5$ & 0.34 & \pm 0.14 & ${ }_{-0.18}^{+0.04}$ & ${ }_{-0.09}^{+0.03}$ & 0.91 & 8.72 \\
$-0.5-0.0$ & 13.6 & \pm 1.0 & ${ }_{-2.4}^{+3.0}$ & ${ }_{-2.1}^{+2.9}$ & 0.98 & 2.30 \\
$0.0-0.5$ & 62.1 & \pm 2.1 & ${ }_{-11.0}^{+9.3}$ & ${ }_{-8.4}^{+9.1}$ & 0.96 & 1.50 \\
$0.5-1.0$ & 115 & \pm 3 & ${ }_{-14}^{+14}$ & ${ }_{-13}^{+14}$ & 0.97 & 1.29 \\
$1.0-1.5$ & 108 & \pm 3 & ${ }_{-14}^{+12}$ & ${ }_{-10}^{+11}$ & 0.96 & 1.22 \\
$1.5-2.0$ & 73.3 & \pm 2.2 & ${ }_{-7.8}^{+7.3}$ & ${ }_{-6.3}^{+7.3}$ & 0.96 & 1.19 \\
$2.0-2.5$ & 20.3 & \pm 1.4 & ${ }_{-2.1}^{+5.6}$ & ${ }_{-1.6}^{+1.7}$ & 0.97 & 1.16 \\
\hline
\end{tabular}

Table 6. The inclusive trijet cross section $d \sigma / d \eta_{\mathrm{LAB}}^{\mathrm{jet}, 3}$ for jets of hadrons in the Breit frame, selected using the $k_{T}$ cluster algorithm in the longitudinally invariant inclusive mode. Other details are as in the caption to Table 1

\begin{tabular}{lclllll}
\hline$\eta_{\mathrm{LAB}}^{\text {jet,3 }}$ & $\begin{array}{c}d \sigma / d \eta_{\mathrm{LAB}}^{\text {jet,3 }} \\
(\mathrm{pb})\end{array}$ & $\delta_{\text {stat }}$ & $\delta_{\text {syst }}$ & $\delta_{\mathrm{ES}}$ & $C_{\mathrm{QED}}$ & $C_{\text {had }}$ \\
\hline$-1.0-0.5$ & 63.7 & \pm 2.3 & ${ }_{-11.0}^{+9.2}$ & ${ }_{-9.3}^{+9.0}$ & 0.96 & 1.32 \\
$-0.5-0.0$ & 112 & \pm 3 & ${ }_{-14}^{+15}$ & ${ }_{-12}^{+15}$ & 0.96 & 1.24 \\
$0.0-0.5$ & 108 & \pm 3 & ${ }_{-15}^{+12}$ & ${ }_{-11}^{+12}$ & 0.96 & 1.25 \\
$0.5-1.0$ & 68.6 & \pm 2.1 & ${ }_{-7.6}^{+8.7}$ & ${ }_{-6.5}^{+7.4}$ & 0.97 & 1.32 \\
$1.0-1.5$ & 31.4 & \pm 1.5 & ${ }_{-4.6}^{+2.3}$ & ${ }_{-2.7}^{+2.1}$ & 0.94 & 1.44 \\
$1.5-2.0$ & 6.21 & \pm 0.67 & ${ }_{-0.67}^{+1.50}$ & ${ }_{-0.61}^{+0.61}$ & 0.93 & 1.50 \\
\hline
\end{tabular}

are generally well described by the NLO QCD predictions. The largest difference is a slightly different slope of the $\eta_{\mathrm{LAB}}^{\text {jet }}$ dependence of the third jet.

\subsection{Cross-section ratio and determination of $\alpha_{s}$}

Figure 4 and Table 9 show the cross-section ratio $R_{3 / 2}$ of the trijet cross section to the dijet cross section, as a function of $Q^{2}$. The correlated systematic and the renormalisation scale uncertainties largely cancel in the ratio. The agreement between the data and NLO predictions is good. The total experimental and theoretical uncertainties are about $5 \%$ and $7 \%$, respectively. These uncertainties are substantially reduced with respect to those of the di- and trijet cross sections. In particular, at low $Q^{2}\left(Q^{2}<100 \mathrm{GeV}^{2}\right)$, 
Table 7. The inclusive dijet cross section $\left(d \sigma / d Q^{2}\right)_{\text {dijet }}$ for jets of hadrons in the Breit frame, selected using the $k_{T}$ cluster algorithm in the longitudinally invariant inclusive mode. Other details are as in the caption to Table 1

\begin{tabular}{lllllll}
\hline$Q^{2}$ & $\left(d \sigma / d Q^{2}\right)_{\text {dijet }}$ & $\delta_{\text {stat }}$ & $\delta_{\text {syst }}$ & $\delta_{\mathrm{ES}}$ & $C_{\mathrm{QED}}$ & $C_{\text {had }}$ \\
$\left(\mathrm{GeV}^{2}\right)$ & $\left(\mathrm{pb} / \mathrm{GeV}^{2}\right)$ & & & & & \\
\hline $10-35$ & 9.70 & \pm 0.10 & ${ }_{-0.94}^{+1.10}$ & ${ }_{-0.76}^{+0.87}$ & 0.97 & 1.18 \\
$35-85$ & 2.50 & \pm 0.04 & ${ }_{-0.20}^{+0.21}$ & ${ }_{-0.18}^{+0.20}$ & 0.95 & 1.16 \\
$85-220$ & 0.649 & \pm 0.011 & ${ }_{-0.044}^{+0.049}$ & ${ }_{-0.041}^{+0.044}$ & 0.96 & 1.15 \\
$220-700$ & 0.104 & \pm 0.002 & ${ }_{-0.006}^{+0.006}$ & ${ }_{-0.006}^{+0.006}$ & 0.94 & 1.12 \\
$700-5000$ & 0.00403 & \pm 0.00014 & ${ }_{-0.00024}^{+0.0015}$ & ${ }_{-0.00014}^{+0.00014}$ & 0.92 & 1.09 \\
\hline
\end{tabular}

Table 8. The inclusive trijet cross section $\left(d \sigma / d Q^{2}\right)$ trijet for jets of hadrons in the Breit frame, selected using the $k_{T}$ cluster algorithm in the longitudinally invariant inclusive mode. Other details are as in the caption to Table 1

\begin{tabular}{lllllll}
\hline $\begin{array}{l}Q^{2} \\
\left(\mathrm{GeV}^{2}\right)\end{array}$ & $\begin{array}{l}\left(d \sigma / d Q^{2}\right)_{\text {trijet }} \\
\left(\mathrm{pb} / \mathrm{GeV}^{2}\right)\end{array}$ & $\delta_{\text {stat }}$ & $\delta_{\text {syst }}$ & $\delta_{\mathrm{ES}}$ & $C_{\mathrm{QED}}$ & $C_{\text {had }}$ \\
\hline $10-35$ & 3.94 & \pm 0.08 & ${ }_{-0.55}^{+0.54}$ & ${ }_{-0.46}^{+0.49}$ & 0.98 & 1.35 \\
$35-85$ & 0.94 & \pm 0.02 & ${ }_{-0.11}^{+0.12}$ & ${ }_{-0.09}^{+0.11}$ & 0.95 & 1.31 \\
$85-220$ & 0.227 & \pm 0.007 & ${ }_{-0.027}^{+0.024}$ & ${ }_{-0.023}^{+0.024}$ & 0.96 & 1.32 \\
$220-700$ & 0.0320 & \pm 0.0013 & ${ }_{-0.0031}^{+0.0036}$ & ${ }_{-0.0026}^{+0.0027}$ & 0.94 & 1.35 \\
$700-5000$ & 0.00112 & \pm 0.00007 & ${ }_{-0.00014}^{+0.00008}$ & ${ }_{-0.00009}^{+0.00008}$ & 0.92 & 1.33 \\
\hline
\end{tabular}

\section{ZEUS}

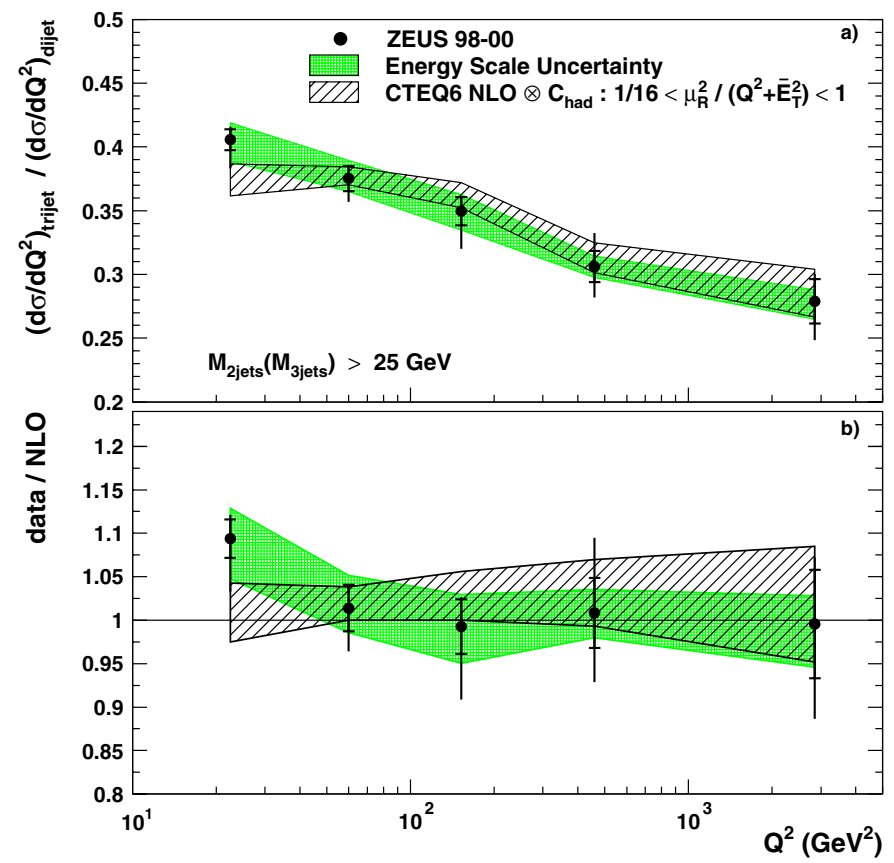

Fig. 4. a The ratio of inclusive trijet to dijet cross sections as a function of $Q^{2}$. The predictions of perturbative QCD in next-to-leading order are compared to the data. $\mathbf{b}$ shows the ratio of the data to the predictions. Other details are as in the caption to Fig. 1

the theoretical uncertainties are reduced by as much as a factor of four. This reduction allows the determination of $\alpha_{s}\left(M_{Z}\right)$ at a much lower $Q^{2}$ than in previous analyses $[14,18]$.
Table 9. The ratio of inclusive trijet to dijet cross sections for jets of hadrons in the Breit frame, selected using the $k_{T}$ cluster algorithm in the longitudinally invariant inclusive mode. Other details are as in the caption to Table 1

\begin{tabular}{lllllll}
\hline $\begin{array}{l}Q^{2} \\
\left(\mathrm{GeV}^{2}\right)\end{array}$ & $R_{3 / 2}$ & $\delta_{\text {stat }}$ & $\delta_{\text {syst }}$ & $\delta_{\mathrm{ES}}$ & $C_{\mathrm{QED}}$ & $C_{\text {had }}$ \\
\hline $10-35$ & 0.406 & \pm 0.008 & ${ }_{-0.027}^{+0.014}$ & ${ }_{-0.017}^{+0.013}$ & 1.01 & 1.14 \\
$35-85$ & 0.375 & \pm 0.010 & ${ }_{-0.019}^{+0.014}$ & ${ }_{-0.010}^{+0.014}$ & 0.99 & 1.02 \\
$85-220$ & 0.350 & \pm 0.011 & ${ }_{-0.031}^{+0.014}$ & ${ }_{-0.015}^{+0.013}$ & 1.00 & 1.15 \\
$220-700$ & 0.306 & \pm 0.013 & ${ }_{-0.022}^{+0.024}$ & ${ }_{-0.009}^{+0.008}$ & 1.01 & 1.21 \\
$700-5000$ & 0.279 & \pm 0.018 & ${ }_{-0.029}^{+0.016}$ & ${ }_{-0.014}^{+0.009}$ & 1.01 & 1.21 \\
\hline
\end{tabular}

The measurement of $R_{3 / 2}$ as a function of $Q^{2}$ was used to determine $\alpha_{s}\left(M_{Z}\right)$ with a method similar to that of a previous ZEUS publication [13]:

- the NLO QCD calculation of $R_{3 / 2}$ was performed using the five sets of proton PDFs of the CTEQ4 A-series $[56]^{3}$. The value of $\alpha_{s}\left(M_{Z}\right)$ used in each partonic cross-section calculation was that associated with the corresponding set of PDFs: $0.110,0.113,0.116,0.119,0.122$;

- for each bin, $i$, in $Q^{2}$, the NLO QCD calculations, corrected for hadronisation effects, were used to parameterise the $\alpha_{s}\left(M_{Z}\right)$ dependence of $R_{3 / 2}$ according to the functional form:

$$
\left[R_{3 / 2}\left(\alpha_{s}\left(M_{Z}\right)\right)\right]^{i}=C_{1}^{i} \cdot \alpha_{s}\left(M_{Z}\right)+C_{2}^{i} \cdot \alpha_{s}^{2}\left(M_{Z}\right),
$$

3 The CTEQ4 PDF was chosen because the CTEQ6 does not provide PDF sets obtained with different $\alpha_{s}\left(M_{Z}\right)$ values and therefore cannot be used for the determination of $\alpha_{s}$. 
where $C_{1}^{i}$ and $C_{2}^{i}$ are fitting parameters. This simple parameterisation gives a good description of the $\alpha_{s}\left(M_{Z}\right)$ dependence of $R_{3 / 2}\left(Q^{2}\right)$ over the entire $\alpha_{s}$ range spanned by the PDF sets;

- a value of $\alpha_{s}\left(M_{Z}\right)$ was then determined in each bin of $Q^{2}$, as well as in the entire $Q^{2}$ region, by a $\chi^{2}$-fit of the measured $R_{3 / 2}\left(Q^{2}\right)$ values using the parameterisation in (1).

This procedure correctly handles the complete $\alpha_{s}$-dependence of the NLO differential cross sections (the explicit dependence coming from the partonic cross sections and the implicit dependence coming from the PDFs) in the fit, while preserving the correlation between $\alpha_{s}$ and the PDFs. Taking into account only the statistical uncertainties on the measured cross-section ratio, $\alpha_{s}\left(M_{Z}\right)$ is determined to be $\alpha_{s}\left(M_{Z}\right)=0.1179 \pm 0.0013$ (stat.).

Fig. 5a shows the sensitivity of the cross-section ratio $R_{3 / 2}$ to the value of $\alpha_{s}$. Figure $5 \mathrm{~b}$ and Table 10 show $\alpha_{s}\left(M_{Z}\right)$ determined in the five bins of $Q^{2}$.

As a cross-check of the extracted value of $\alpha_{s}\left(M_{Z}\right)$, the fit procedure was repeated by using the three sets of the MRST99 PDF corresponding to $\alpha_{s}\left(M_{Z}\right)$ equal to 0.1125 , 0.1175 , and 0.1225 . The result is

$$
\alpha_{s}\left(M_{Z}\right)=0.1178 \pm 0.0010 \text { (stat.) }
$$

\section{ZEUS}

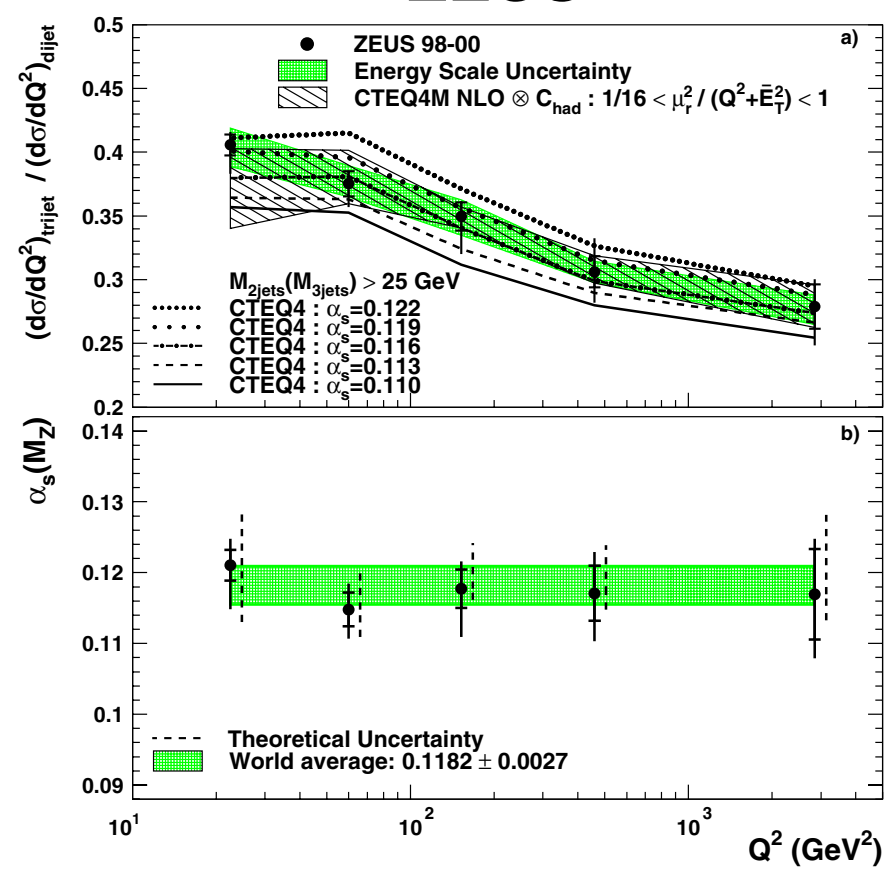

Fig. 5. a The ratio of inclusive trijet to dijet cross sections as a function of $Q^{2}$. The predictions of perturbative QCD in nextto-leading order using five sets of CTEQ4 PDF are compared to the data. $\mathbf{b}$ shows the $\alpha_{s}\left(M_{Z}\right)$ values determined from the ratio of inclusive trijet to dijet cross sections in different regions of $Q^{2}$. The shaded band indicates the current world average value of $\alpha_{s}\left(M_{Z}\right)$. The inner error bars represent the statistical uncertainty of the data. The outer error bars show the statistical and systematic uncertainties added in quadrature. The dashed error bars display the theoretical uncertainties
Table 10. The $\alpha_{s}\left(M_{Z}\right)$ values as determined in this analysis. The statistical $\left(\delta_{\text {stat }}\right)$, experimental $\left(\delta_{\exp }\right)$ and theoretical $\left(\delta_{\text {theo }}\right)$ systematic uncertainties are shown separately

\begin{tabular}{lllll}
\hline $\begin{array}{l}Q^{2} \\
\left(\mathrm{GeV}^{2}\right)\end{array}$ & $\alpha_{s}\left(M_{Z}\right)$ & $\delta_{\text {stat }}$ & $\delta_{\exp }$ & $\delta_{\text {theo }}$ \\
\hline $10-35$ & 0.1210 & \pm 0.0022 & ${ }_{-0.0058}^{+0.0031}$ & ${ }_{-0.0080}^{+0.0074}$ \\
$35-85$ & 0.1148 & \pm 0.0024 & ${ }_{-0.0033}^{+0.0028}$ & ${ }_{-0.0039}^{+0.0056}$ \\
$85-220$ & 0.1178 & \pm 0.0027 & ${ }_{-0.0063}^{+0.0027}$ & ${ }_{-0.0016}^{+0.0064}$ \\
$220-700$ & 0.1171 & \pm 0.0039 & ${ }_{-0.0056}^{+0.0043}$ & ${ }_{-0.0023}^{+0.0068}$ \\
$700-5000$ & 0.1170 & \pm 0.0064 & ${ }_{-0.0064}^{+0.0045}$ & ${ }_{-0.0037}^{+0.011}$ \\
\hline $10-5000$ & 0.1179 & \pm 0.0013 & ${ }_{-0.0046}^{+0.0028}$ & ${ }_{-0.0046}^{+0.0064}$ \\
\hline
\end{tabular}

In addition, the NLO QCD analysis used to obtain the ZEUS-S PDF [58] was repeated to obtain a set of five PDFs corresponding to the values of $\alpha_{s}\left(M_{Z}\right): 0.115,0.117$, $0.119,0.121,0.123$. These sets were used in the current analysis yielding $\alpha_{s}\left(M_{Z}\right)=0.1191 \pm 0.0010$ (stat.), in good agreement with the other determinations.

The experimental and theoretical uncertainties of the extracted value of $\alpha_{s}\left(M_{Z}\right)$ were evaluated by repeating the analysis above for each systematic check, as described in Sect.6. The main contributions to the experimental systematic uncertainty were:

- jet pseudorapidity cut $\left({ }_{-1.5 \%}^{+1 \%}\right)$;

- jet transverse energy and invariant mass cuts $\left({ }_{-2 \%}^{+0.5 \%}\right)$;

- use of different parton shower model $(-2 \%)$;

- the absolute energy scale of the CAL $\left({ }_{-2.5 \%}^{+2 \%}\right)$.

The main contributions to the theoretical uncertainty are:

- uncertainties in the proton PDFs $\left({ }_{-2 \%}^{+1.5 \%}\right)$;

- uncertainties in the correction factor, $C_{\mathrm{had}}(+2 \%)$;

- uncertainties due to terms beyond $N L O\left(_{-3.5 \%}^{+5 \%}\right)$.

The value of $\alpha_{s}\left(M_{Z}\right)$ as determined from the measurements of $R_{3 / 2}$ is therefore:

$\alpha_{s}\left(M_{Z}\right)=0.1179 \pm 0.0013(\text { stat. })_{-0.0046}^{+0.0028}(\text { exp. })_{-0.0046}^{+0.0064}($ th. $)$.

The result is in good agreement with recent determinations at HERA $[10,12-19]$ and the current world average of $\alpha_{s}\left(M_{Z}\right)=0.1182 \pm 0.0027[60,61]$.

\section{Summary}

Differential dijet and trijet cross sections have been measured with high precision in neutral current deep inelastic scattering for $10<Q^{2}<5000 \mathrm{GeV}^{2}$ at HERA using the ZEUS detector. The inclusive trijet cross section has been measured as a function of $E_{T, B}^{\mathrm{jet}}, \eta_{\mathrm{LAB}}^{\mathrm{jet}}$ and $Q^{2}$. The ratio $R_{3 / 2}$ of the trijet and dijet cross sections has been measured as a function of $Q^{2}$. The predictions of perturbative QCD calculations in next-to-leading order give a good description of the dijet and trijet cross sections and the cross-section ratio $R_{3 / 2}$ over the whole range 
of $Q^{2}$. The cancellation of uncertainties in the ratio, in particular those from theory, allow the extraction of $\alpha_{s}$ with good precision down to $Q^{2}$ of $10 \mathrm{GeV}^{2}$. The value of the strong coupling constant $\alpha_{s}$ was measured to be $\alpha_{s}\left(M_{Z}\right)=0.1179 \pm 0.0013$ (stat. $)_{-0.0046}^{+0.0028}(\text { exp. })_{-0.0046}^{+0.0064}($ th. $)$, in good agreement with the current world average value and previous determinations of $\alpha_{s}\left(M_{Z}\right)$ at HERA.

Acknowledgements. We thank the DESY Directorate for their strong support and encouragement. The remarkable achievements of the HERA machine group were essential for the successful completion of this work and are greatly appreciated. The design, construction and installation of the ZEUS detector has been made possible by the effort of many people who are not listed as authors. We would like to thank Z. Nagy for useful discussions.

\section{References}

1. UA1 Coll., G. Arnison et al., Phys. Lett. B 158, 494 (1985)

2. UA2 Coll., J.A. Appel et al., Z. Phys. C 30, 341 (1986)

3. CMOR Coll., A.L. S. Angelis, Nucl. Phys. B $\mathbf{3 0 3}$, 569 (1988)

4. Axial Field Spectrometer Coll., T. Akesson et al., Z. Phys. C 32, 317 (1986)

5. D0 Coll., B. Abbott et al., Phys. Rev. Lett. 86, 1955 (2001)

6. D0 Coll., S. Abachi et al., Phys. Rev. D 53, 6000 (1996)

7. P. Lutz, in: Proceedings of the 31st Rencontres de Moriond: QCD and High-Energy Hadronic Interactions, Les Arcs, France, 23-30 Mar 1996, pp. 223-228

8. Z. Nagy, Z. Trocsanyi, Nucl. Phys. Proc. Suppl. 74, 44 (1999)

9. ZEUS Coll., J. Breitweg et al., Phys. Lett. B 443, 394 (1998)

10. H1 Coll., C. Adloff et al., Eur. Phys. J. C 6, 575 (1999)

11. H1 Coll., C. Adloff et al., Phys. Lett. B 515, 17 (2001)

12. ZEUS Coll., M. Derrick et al., Phys. Lett. B 363, 201 (1995)

13. ZEUS Coll., J. Breitweg et al., Phys. Lett. B 507, 70 (2001)

14. ZEUS Coll., S. Chekanov et al., Phys. Lett. B 547, 164 (2002)

15. ZEUS Coll., S. Chekanov et al., Phys. Lett. B 558, $41(2003)$

16. H1 Coll., C. Adloff et al., Eur. Phys. J. C 19, 289 (2001)

17. H1 Coll., C. Adloff et al., Eur. Phys. J. C 21, 33 (2001)

18. ZEUS Coll., S. Chekanov et al., Nucl. Phys. B 700, 3 (2004)

19. ZEUS Coll., S. Chekanov et al., Phys. Lett. B 560, 7 (2003)

20. ZEUS Coll., M. Derrick et al., Phys. Lett. B 293, 465 (1992)

21. ZEUS Coll., U. Holm (ed.), The ZEUS Detector. Status Report (unpublished), DESY (1993), available on http://www-zeus.desy.de/bluebook/bluebook.html

22. N. Harnew et al., Nucl. Instrum. Methods A 279, 290 (1989)

23. B. Foster et al., Nucl. Phys. Proc. Suppl. B 32, 181 (1993)

24. B. Foster et al., Nucl. Instrum. Methods A 338, 254 (1994)

25. M. Derrick et al., Nucl. Instrum. Methods A 309, 77 (1991)

26. A. Andresen et al., Nucl. Instrum. Methods A 309, 101 (1991)
27. A. Caldwell et al., Nucl. Instrum. Methods A 321, 356 (1992)

28. A. Bernstein et al., Nucl. Instrum. Methods A 336 , 23 (1993)

29. J. Andruszków et al., Preprint DESY-92-066, DESY (1992)

30. ZEUS Coll., M. Derrick et al., Z. Phys. C 63, 391 (1994)

31. J. Andruszków et al., Acta Phys. Pol. B 32, 2025 (2001)

32. ZEUS Coll., J. Breitweg et al., Z. Phys. C 74, 207 (1997)

33. N. Krumnack, Ph.D. Thesis, University of Hamburg (2004)

34. L. Li, Ph.D. Thesis, University of WisconsinMadison (2005)

35. ZEUS Coll., J. Breitweg et al., Eur. Phys. J. C 11, 427 (1999)

36. S. Bentvelsen, J. Engelen, P. Kooijman, in: Proceedings of the Workshop on Physics at HERA, edited by W. Buchmüller, G. Ingelman, Hamburg, Germany, DESY (1992), Vol. 1, p. 23

37. F. Jacquet, A. Blondel, in: Proceedings of the Study for an $e p$ Facility for Europe, edited by U. Amaldi, Hamburg, Germany (1979), p. 391; also in preprint DESY 79/48

38. ZEUS Coll., S. Chekanov et al., Eur. Phys. J. C 23, $13(2002)$

39. S.Catani et al., Nucl. Phys. B 406, 187 (1993)

40. S.D. Ellis, D.E. Soper, Phys. Rev. D 48, 3160 (1993)

41. R.P. Feynman, Photon-Hadron Interactions (Benjamin, New York, 1972)

42. K.H. Streng, T.F. Walsh, P.M. Zerwas, Z. Phys. C 2, 237 (1979)

43. ZEUS Coll., S. Chekanov et al., Preprint hep-ex/0404033 (2004)

44. J.E. Huth et al., Research Directions for the Decade, in: Proceedings of the Summer Study on High Energy Physics, 1990, edited by E.L. Berger (World Scientific, 1992), p. 134; also in preprint FERMILAB-CONF-90-249-E

45. L. Lönnblad, Comput. Phys. Commun. 71, 15 (1992)

46. G. Ingelman, A. Edin, J. Rathsman, Comput. Phys. Commun. 101, 108 (1997)

47. A. Kwiatkowski, H. Spiesberger, H.-J. Möhring, Comput. Phys. Commun. 69, 155 (1992); also in: Proceedings of the Workshop Physics at HERA, 1991, DESY, Hamburg

48. K. Charchula, G.A. Schuler, H. Spiesberger, Comput. Phys. Commun. 81, 381 (1994)

49. G. Gustafson, U. Pettersson, Nucl. Phys. B 306, 746 (1988)

50. B. Andersson et al., Phys. Rep. 97, 31 (1983)

51. M. Bengtsson, T. Sjöstrand, Comput. Phys. Commun. 46, 43 (1987)

52. T. Sjöstrand, Comput. Phys. Commun. 82, 74 (1994)

53. R. Brun et al., GEANT3, Technical Report CERNDD/EE/84-1, CERN (1987)

54. Z. Nagy, Z. Trocsanyi, Phys. Rev. Lett. 87, 082001 (2001)

55. J. Pumplin et al., Preprint hep-ph/0201195 (2002)

56. H.L. Lai et al., Phys. Rev. D 55, 1280 (1997)

57. A.D. Martin et al., Eur. Phys. J. C 14, 133 (2000)

58. ZEUS Coll., S. Chekanov et al., Phys. Rev. D 67, 012007 (2003)

59. S. Catani, M.H. Seymour, Nucl. Phys. B 485, 291 (1997)

60. S. Bethke, J. Phys. G 26, R27 (2000)

61. Updated in S. Bethke, Talk given at 7th DESY Workshop on Elementary Particle Theory: Loops and Legs in Quantum Field Theory, Zinnowitz, Germany, 25-30 Apr 2004, preprint hep-ex/0407021 (2004) 